\title{
Ventricular kinetic energy may provide a novel noninvasive way to assess ventricular performance in patients with repaired tetralogy of Fallot
}

Daniel Jeong, MD, MS, ${ }^{\mathrm{a}, \mathrm{b}}$ Petros V. Anagnostopoulos, MD, ${ }^{\mathrm{c}}$ Alejandro Roldan-Alzate, PhD,

Shardha Srinivasan, MD, ${ }^{\mathrm{d}}$ Mark L. Schiebler, MD, ${ }^{\mathrm{a}}$ Oliver Wieben, $\mathrm{PhD},{ }^{\mathrm{a}, \mathrm{e}}$ and

Christopher J. François, MD

\section{ABSTRACT}

Objective: Ventricular kinetic energy measurements may provide a novel imaging biomarker of declining ventricular efficiency in patients with repaired tetralogy of Fallot. Our purpose was to assess differences in ventricular kinetic energy with 4-dimensional flow magnetic resonance imaging between patients with repaired tetralogy of Fallot and healthy volunteers.

Methods: Cardiac magnetic resonance, including 4-dimensional flow magnetic resonance imaging, was performed at rest in 10 subjects with repaired tetralogy of Fallot and 9 healthy volunteers using clinical 1.5T and 3T magnetic resonance imaging scanners. Right and left ventricular kinetic energy $\left(\mathrm{KE}_{\mathrm{RV}}\right.$ and $\left.\mathrm{KE}_{\mathrm{LV}}\right)$, main pulmonary artery flow $\left(\mathrm{Q}_{\mathrm{MPA}}\right)$, and aortic flow $\left(\mathrm{Q}_{\mathrm{AO}}\right)$ were quantified using 4-dimensional flow magnetic resonance imaging data. Right and left ventricular size and function were measured using standard cardiac magnetic resonance techniques. Differences in peak systolic $\mathrm{KE}_{\mathrm{RV}}$ and $\mathrm{KE}_{\mathrm{LV}}$ in addition to the $\mathrm{Q}_{\mathrm{MPA}} / \mathrm{KE}_{\mathrm{RV}}$ and $\mathrm{Q}_{\mathrm{AO}} / \mathrm{KE}_{\mathrm{LV}}$ ratios between groups were assessed. Kinetic energy indices were compared with conventional cardiac magnetic resonance parameters.

Results: Peak systolic $\mathrm{KE}_{\mathrm{RV}}$ and $\mathrm{KE}_{\mathrm{LV}}$ were higher in patients with repaired tetralogy of Fallot $(6.06 \pm 2.27 \mathrm{~mJ}$ and $3.55 \pm 2.12 \mathrm{~mJ}$, respectively) than in healthy volunteers $(5.47 \pm 2.52 \mathrm{~mJ}$ and $2.48 \pm 0.75 \mathrm{~mJ}$, respectively), but were not statistically significant $\left(P=.65\right.$ and $P=.47$, respectively). The $\mathrm{Q}_{\mathrm{MPA}} / \mathrm{KE}_{\mathrm{RV}}$ and $\mathrm{Q}_{\mathrm{AO}} / \mathrm{KE}_{\mathrm{LV}}$ ratios were lower in patients with repaired tetralogy of Fallot (7.53 $\pm 5.37 \mathrm{~mL} /[$ cycle $\mathrm{mJ}]$ and $9.65 \pm 6.61 \mathrm{~mL} /[$ cycle $\mathrm{mJ}]$, respectively) than in healthy volunteers $(19.33 \pm 18.52 \mathrm{~mL} /[$ cycle $\mathrm{mJ}]$ and $35.98 \pm 7.66 \mathrm{~mL} /[$ cycle $\mathrm{mJ}]$, respectively; $P<.05)$. $\mathrm{Q}_{\mathrm{MPA}} / \mathrm{KE}_{\mathrm{RV}}$ and $\mathrm{Q}_{\mathrm{AO}} / \mathrm{KE}_{\mathrm{LV}}$ were weakly correlated to ventricular size and function.

Conclusions: Greater ventricular kinetic energy is necessary to generate flow in the pulmonary and aortic circulations in repaired tetralogy of Fallot. Quantification of ventricular kinetic energy in patients with repaired tetralogy of Fallot is a new observation. Future studies are needed to determine whether changes in ventricular kinetic energy can provide earlier evidence of ventricular dysfunction and guide future medical and surgical interventions. (J Thorac Cardiovasc Surg 2015;149:1339-47)

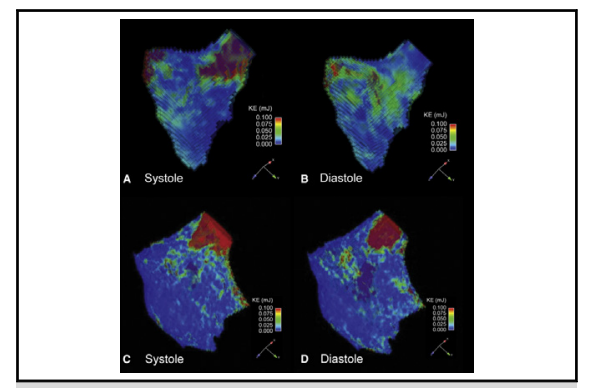

RV kinetic energy is a potentially new marker for impaired RV function in rTOF.

Central Message

Greater kinetic energy is required to generate flow in rTOF, which may provide earlier evidence of ventricular dysfunction.

\section{Perspective}

Quantification of ventricular kinetic energy in rTOF is a new observation, with a complex relationship between ventricular kinetic energy and great vessel flow. Greater energy losses in rTOF, indicative of increased inefficiency, is a novel method of monitoring declining cardiac function and may provide earlier evidence for intervention than current standard indices.

See Editorial Commentary page 1348
From the Department of Radiology, ${ }^{\text {a }}$ University of Wisconsin-Madison, Madison, Wis; Department of Radiology, ${ }^{\mathrm{b}}$ Moffitt Cancer Center, Tampa, Fla; and Departments of Surgery, ${ }^{\mathrm{c}}$ Pediatrics, ${ }^{\mathrm{d}}$ and Medical Physics, ${ }^{\mathrm{e}}$ University of Wisconsin-Madison, Madison, Wis.

Supported by National Institutes of Health Grant R01HL07226 and Department of Radiology Research and Development Fund.

Received for publication June 9, 2014; revisions received Oct 15, 2014; accepted for publication Nov 29, 2014; available ahead of print Jan 23, 2015.
Address for reprints: Christopher J. François, MD, Department of Radiology, University of Wisconsin-Madison, School of Medicine and Public Health, 600 Highland Ave, Madison, WI 53562 (E-mail: cfrancois@uwhealth.org). $0022-5223 / \$ 36.00$

Copyright $(2015$ by The American Association for Thoracic Surgery http://dx.doi.org/10.1016/j.jtcvs.2014.11.085 


$$
\begin{array}{ll}
\text { Abbreviations and Acronyms } \\
\mathrm{BSA} & =\text { body surface area } \\
\mathrm{CMR} & =\text { cardiac magnetic resonance } \\
\mathrm{EDVI} & =\text { end-diastolic volume index } \\
\mathrm{EF} & =\text { ejection fraction } \\
\mathrm{ESVI} & =\text { end-systolic volume index } \\
\mathrm{4D} & =4 \text {-dimensional } \\
\mathrm{IV} & =\text { intravenous } \\
\mathrm{KE} & =\text { kinetic energy } \\
\mathrm{KE} & =\text { left ventricular kinetic energy } \\
\mathrm{KE} & =\text { right ventricular kinetic energy } \\
\mathrm{LV} & =\text { left ventricular } \\
\mathrm{MRI} & =\text { magnetic resonance imaging } \\
\mathrm{PA} & =\text { pulmonary artery } \\
\mathrm{PC} \mathrm{VIPR} & =\text { vastly undersampled isotropic } \\
& \text { projection reconstruction } \\
\mathrm{Q}_{\mathrm{AO}} & =\text { aortic flow } \\
\mathrm{Q}_{\mathrm{MPA}} & =\text { main pulmonary artery flow } \\
\mathrm{rTOF} & =\text { repaired tetralogy of Fallot } \\
\mathrm{RV} & =\text { right ventricular } \\
\mathrm{TOF} & =\text { tetralogy of Fallot }
\end{array}
$$

Without surgical intervention, the natural history of tetralogy of Fallot (TOF) can lead to mortality rates of $25 \%$ in infants with severe obstruction within the first year of life and up to $95 \%$ by age 40 years. ${ }^{1}$ TOF is the most common cyanotic congenital heart disease accounting for $9 \%$ to $14 \%$ of all congenital cardiovascular defects. ${ }^{2}$ With advancements in technology and surgical technique, most patients now undergo corrective surgical repair early in life and live into adulthood. Surgical correction of TOF typically involves ventricular septal defect closure, relief of right ventricular (RV) outflow tract obstruction, disruption of the pulmonary valve that results in pulmonary regurgitation, and placement of an outflow patch. ${ }^{3}$ Expected postoperative pulmonary regurgitation has been associated with progressive RV dilatation and ventricular dysfunction. ${ }^{4}$ Alterations in hemodynamics that frequently occur after repair ultimately contribute to poor long-term outcomes, including progressive exercise intolerance, ventricular arrhythmia, and sudden cardiac death. ${ }^{5}$

Cardiac magnetic resonance (CMR) has become the gold standard to monitor patients with repaired TOF (rTOF). Volumetric and functional CMR parameters are used to guide the decision of when to perform pulmonary valve replacement to protect from the long-term sequelae of chronic pulmonary insufficiency. ${ }^{6}$ Monitoring with conventional CMR relies on detecting underlying morphologic changes, such as RV dilatation, to signify dysfunction. The newer 4-dimensional (4D) flow magnetic resonance imaging (MRI) technique can detect abnormal $\mathrm{RV}$ diastolic flow patterns in rTOF even when RV volumes or function is not substantially abnormal. ${ }^{7,8}$ Although pulmonary valve replacement results in reversal of RV dilation, the risk of cardiac death and arrhythmias may not be averted. ${ }^{9}$ More sensitive markers of RV dysfunction could help guide therapy and timing of pulmonary valve insertion.

Ventricular kinetic energy (KE) measurements provide a novel method of monitoring cardiac function ${ }^{10,11}$ and may provide an earlier imaging biomarker of declining ventricular efficiency in patients with rTOF compared with conventional measurements of ventricular size and function, which are based on morphologic changes. Previous studies have demonstrated the feasibility of calculating KE noninvasively with 4D flow MRI. ${ }^{12,13}$ In addition, 4D flow MRI provides aortic and main pulmonary artery (PA) flow data allowing the relationship of $\mathrm{KE}$ and the generated ventricular outflow to be evaluated. Traditionally, the ventricular-vascular relationship or coupling is described by elastance of each component, derived from pressure-volume loops. ${ }^{14}$ With the availability of 4D flow MRI, an analogous ventricular KE and vascular outflow relationship can be studied. To our knowledge, there have been no previous studies that have examined ventricular KE measurements in patients with rTOF. The purpose of this study was to assess differences in ventricular KE between rTOF and healthy volunteers using 4D flow MRI.

\section{MATERIALS AND METHODS \\ Subjects}

This single-center prospective cohort study was approved by the university Institutional Review Board. Data were acquired in compliance with all applicable Health Insurance Portability and Accountability Act regulations. Written informed consent was obtained from all subjects aged 18 years or more. For subjects aged 18 years or less, written informed consent was obtained from parents or legal guardians and assent obtained from subjects aged 6 years or more. Ten patients with rTOF (age $20.6 \pm 12.2$ years) and 9 healthy volunteers (age $38.9 \pm 15.1$ years) were included in this study. Healthy volunteers responded to public recruitment advertisements and were selected to participate after passing a health screening for cardiovascular disease. All subjects underwent CMR examinations after obtaining appropriate consent/assent.

\section{Cardiac Magnetic Resonance}

In subjects with rTOF, the clinically indicated CMR performed to measure ventricular size and function was followed by an investigational 4D flow MRI acquisition. Subjects with rTOF were scanned at $1.5 \mathrm{~T}$ (HDx, GE Healthcare, Waukesha, Wis) or 3.0T (MR750, GE Healthcare), depending on the clinical availability of the scanners at the time of the examination and need for sedation. In healthy volunteers, 4D flow MRI and 2-dimensional cine balanced steady-state free precession were performed on 3.0T scanners (MR750, GE Healthcare).

Details of the 4D flow MRI sequence, phase contrast with vastly undersampled isotropic projection reconstruction (PC VIPR), have been reported. $^{7,15}$ Briefly, 4D flow MRI parameters were as follows: field of view $=260$ to $320 \mathrm{~mm}$, spatial resolution $=1.3 \mathrm{~mm}$ isotropic, repetition time $=8.8$ to $10.9 \mathrm{~ms}(1.5 \mathrm{~T})$ and 6.2 to $3.7 \mathrm{~ms} \mathrm{(3.0T),} \mathrm{echocardiography}$ time $=2.8$ to $3.7 \mathrm{~ms}(1.5 \mathrm{~T})$ and 2.0 to $2.2 \mathrm{~ms}$ (3.0T), velocity 
encoding $=40$ to $400 \mathrm{~cm} / \mathrm{s}$ (adjusted to maximize image quality while minimizing velocity aliasing artifact), scan time $=9$ to 17 minutes using respiratory and retrospective electrocardiogram gating. Nominal temporal resolution was 35 to $44 \mathrm{~ms}$ at $1.5 \mathrm{~T}$ and $25-27 \mathrm{~ms}$ at $3.0 \mathrm{~T}$. $4 \mathrm{D}$ flow MRI was performed after intravenous (IV) administration of 0.1 $\mathrm{mmol} / \mathrm{kg}$ gadobenate dimeglumine (Bracco Diagnostics, Princeton, NJ) in all healthy volunteers and 7 subjects with rTOF to improve signal-tonoise performance. ${ }^{16}$ IV contrast was not given to 3 subjects with rTOF (poor renal function $n=1$, inability to obtain IV access $n=2$ ). Although many of the subjects with rTOF had previous surgeries, no significant postoperative artifacts were encountered that would have affected local signal to noise or flow measurements.

Subjects and healthy volunteers underwent 2-dimensional cine balanced steady-state free precession imaging oriented along left ventricular (LV) short axis to obtain clinical CMR values for functional assessment. MRI parameters were as follows: field of view $=36 \times 29$ to $36 \mathrm{~cm}$, repetition time $3.2 \mathrm{~ms}$, echo time 1.1 to $1.2 \mathrm{~ms}$, flip angle $45^{\circ}$, matrix $=256 \times 192$, bandwidth $90.9 \mathrm{kHz}$, views per segment 12 to 16 , slice thickness 6 to $8 \mathrm{~mm}$, and slice spacing $0 \mathrm{~mm}$.

\section{Postprocessing}

Segmentation of the RV and LV was conducted using Mimics (Materialise, Leuven, Belgium). Time-averaged magnitude images derived from the PC VIPR data set were used for segmentation. ${ }^{17}$ Segmentation directly applied to the intrinsic magnitude images allowed for seamless integration of the segmented RV and LV with the accompanying 4D flow MRI velocity data. Using a time-averaged sequence allowed for a single segmentation to be applied to the average of the velocity data throughout the cardiac cycle.

Ensight (CEI, Apex, NC) was used for 4D flow MRI visualization and reconstruction of 2-dimensional planes orthogonal to the direction of flow through the ascending aorta and main PA, which provided PA and aortic flow $\left(\mathrm{Q}_{\mathrm{MPA}}\right.$ and $\left.\mathrm{Q}_{\mathrm{AO}}\right)$. Customized MATLAB (The MathWorks, Inc, Natick, Mass) routines were used to calculate RV and $\mathrm{LV} \mathrm{KE}\left(\mathrm{KE}_{\mathrm{RV}}\right.$ and $\mathrm{KE}_{\mathrm{LV}}$, respectively).

$\mathrm{RV}$ and LV end-diastolic volume index (EDVI), end-systolic volume index (ESVI), and ejection fraction (EF) were determined from cine balanced steady-state free precession acquisitions. Volumetric postprocessing was performed on all subjects and healthy volunteers using Report Card software (GE Healthcare) by the same board-certified radiologist.

\section{Kinetic Energy Calculation}

The KE of a voxel of blood was calculated using the equation $\mathrm{KE}=\frac{1}{2}$ $m v^{2}$, where the mass $(m)$ represents the voxel volume multiplied by the density of blood $(1.05 \mathrm{~g} / \mathrm{mL})^{18}$ and the velocity $(v)$ of each voxel was determined from $4 \mathrm{D}$ flow MRI. $\mathrm{KE}_{\mathrm{RV}}$ and $\mathrm{KE}_{\mathrm{LV}}$ were determined from the sum of the KE of the voxels within the segmented RV or LV blood pool, respectively, at each phase of the cardiac cycle. In the KE equation and concurrent with previous studies, the directionality component of velocity is removed because velocity is squared. ${ }^{12}$ Therefore, blood flowing in opposite directions within the ventricle would lead to additive KE values. ${ }^{12} \mathrm{KE}$ values were calculated in millijoules and measured at 20 phases throughout the cardiac cycle.

\section{Data Analysis}

Statistical analyses were conducted using SPSS version 20 (SPSS Inc, Chicago, Ill). Differences in peak systolic $\mathrm{KE}_{\mathrm{RV}}$ and $\mathrm{KE}_{\mathrm{LV}}$ in addition to the $\mathrm{Q}_{\mathrm{MPA}} / \mathrm{KE}_{\mathrm{RV}}$ peak systolic and $\mathrm{Q}_{\mathrm{AO}} / \mathrm{KE}_{\mathrm{LV}}$ peak systolic ratios between rTOF and healthy volunteer groups were assessed using the Wilcoxon rank-sum test. The $\mathrm{Q}_{\mathrm{MPA}} / \mathrm{KE}_{\mathrm{RV}}$ peak systolic and $\mathrm{Q}_{\mathrm{AO}} / \mathrm{KE}_{\mathrm{LV}}$ peak systolic ratios were measured as a marker of ventricular-vascular efficiency or ventricular KE loss. This is a novel ratio that allows quantitative evaluation of the relationship between ventricular outflow and $\mathrm{KE}$. $\mathrm{KE}_{\mathrm{RV}}$ and $\mathrm{Q}_{\mathrm{MPA}} / \mathrm{KE}_{\mathrm{RV}}$ were compared with the RV EF, EDVI, and ESVI using the Pearson correlation coefficient. $\mathrm{KE}_{\mathrm{LV}}$ and $\mathrm{Q}_{\mathrm{AO}} / \mathrm{KE}_{\mathrm{LV}}$ were also compared with the LVEF, EDVI, and ESVI using the Pearson correlation coefficient.

\section{RESULTS}

Ten patients with rTOF (age $20.6 \pm 12.2$ years, 5 female) and 9 healthy volunteers (age $38.9 \pm 15.1$ years, 3 female) were included in this study. Characteristics of the rTOF group are summarized in Table 1 . A total of 7 of the 10 patients with rTOF had transannular patch correction. The type of repair in the other 3 patients was not available. All subjects with rTOF underwent surgical correction when they were aged less than 2 years. None of the patients had significant residual ventricular septal defects, and all had some degree of pulmonary insufficiency. Seven subjects with rTOF were symptomatic at the time of CMR, including fatigue, palpitations, dyspnea, or chest discomfort.

Figure 1 shows the distribution of $\mathrm{RV}_{\mathrm{KE}}$ during systole and early diastole in a healthy volunteer and a subject with rTOF. During systole in healthy volunteers, the focal red signal in Figure 1 shows the highest $\mathrm{KE}$ at the RV outflow tract. During diastole, the highest KE was seen along the RV inner curvature, between the tricuspid valve

TABLE 1. Characteristics of subjects with repaired tetralogy of Fallot

\begin{tabular}{|c|c|c|c|c|c|c|c|}
\hline Subject & $\begin{array}{l}\text { Age at time } \\
\text { of CMR (y) }\end{array}$ & Gender & BSA $\left(\mathbf{m}^{2}\right)$ & Type of repair & Prior shunt & $\begin{array}{c}\text { Age of } \\
\text { last repair }\end{array}$ & Symptoms at time of CMR \\
\hline 1 & 7 & $\mathrm{~F}$ & 0.98 & Transannular patch & N/A & $2 \mathrm{mo}$ & None \\
\hline 2 & 9 & M & 0.96 & $\begin{array}{l}\text { Transannular patch and } \\
\text { pulmonary valvectomy }\end{array}$ & Blalock-Taussig & $6 \mathrm{mo}$ & Dyspnea on exertion \\
\hline 3 & 11 & $\mathrm{~F}$ & 1.17 & Transannular patch & Blalock-Taussig $\times 2$ & $8 \mathrm{mo}$ & Palpitations, chest discomfort \\
\hline 4 & 13 & $\mathrm{~F}$ & 1.51 & Transannular patch & N/A & $6 \mathrm{mo}$ & Palpitations \\
\hline 5 & 15 & $\mathrm{~F}$ & 1.59 & Transannular patch & Blalock-Taussig & $2 \mathrm{y}$ & None \\
\hline 6 & 17 & $\mathrm{~F}$ & 1.62 & Transannular patch & N/A & $2 \mathrm{y}$ & Fatigue \\
\hline 7 & 19 & M & 1.90 & Transannular patch & N/A & $10 \mathrm{mo}$ & None \\
\hline 8 & 34 & M & 2.17 & N/A & Waterston & $16 \mathrm{y}$ & Palpitations \\
\hline 9 & 38 & M & 2.31 & N/A & Waterston & N/A & Palpitations \\
\hline 10 & 43 & $\mathrm{M}$ & 1.71 & N/A & Waterston & N/A & Shortness of breath \\
\hline
\end{tabular}

$C M R$, Cardiac magnetic resonance; $B S A$, body surface area; $N / A$, not available. 


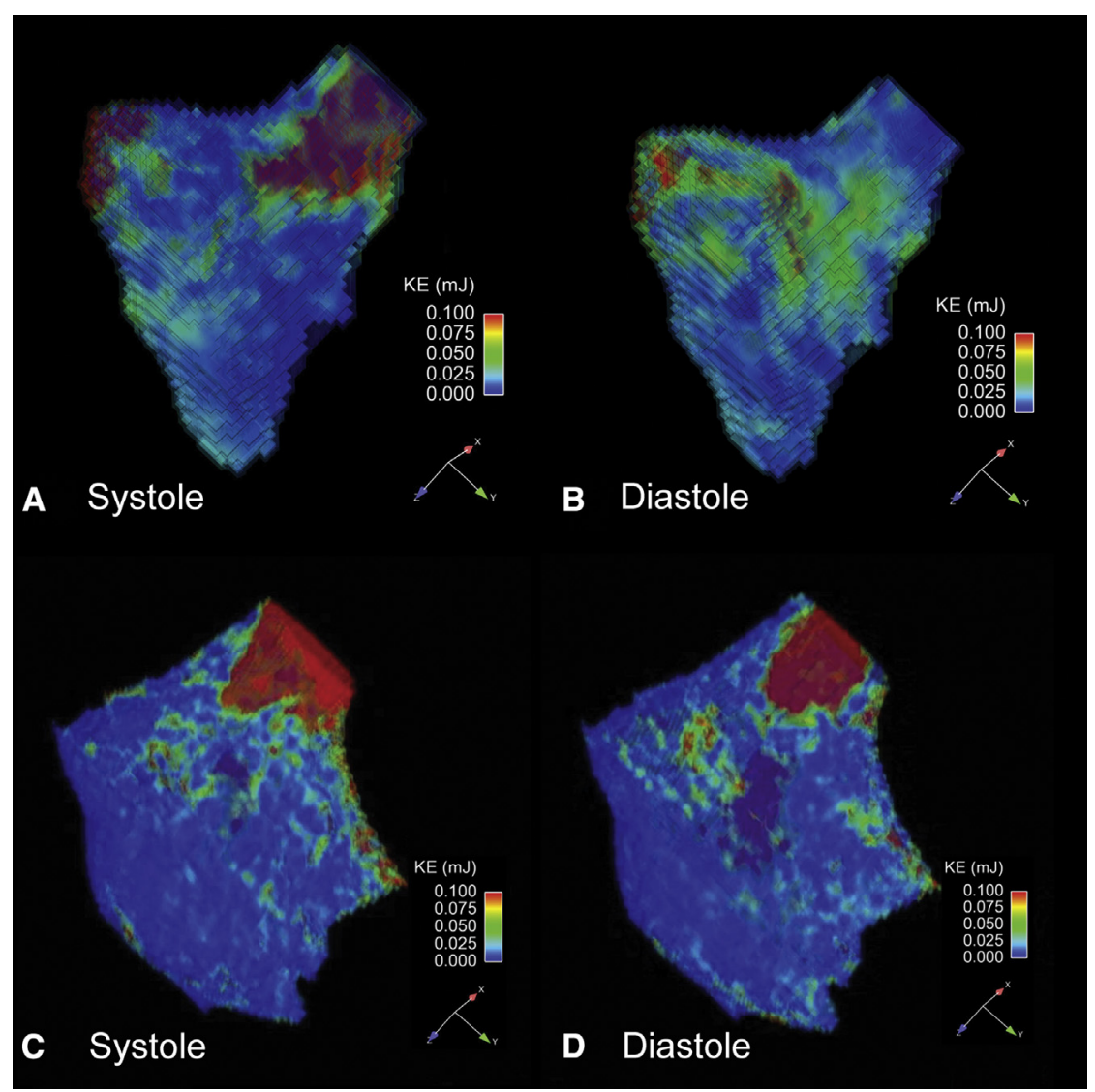

FIGURE 1. A and B, Healthy volunteer RV KE maps in a long-axis orientation. The KE values of each voxel of blood in the segmented plane where the highest KE values are marked in red. Note that highest KE values are seen along the RV outflow tract during systole and along the RV inner curvature during diastole. C and D, rTOF RV KE maps oriented along the RV outflow tract. Note that highest KE values are seen along the RV outflow tract during systole and diastole because of the presence of pulmonic regurgitation in this patient. $K E$, Kinetic energy.

and the RV outflow tract. This distribution of $R V_{\mathrm{KE}}$ was typical for the healthy volunteer group. In those with rTOF, the highest KE was seen along the RV outflow tract in both diastole and systole because of the presence of pulmonic regurgitation. This distribution of $\mathrm{RV}_{\mathrm{KE}}$ was typical for the rTOF group.

Table 2 summarizes volumetric CMR measurements, ventricular KE calculations, and flow measurements. The mean peak systolic $\mathrm{KE}_{\mathrm{RV}}$ in patients with rTOF, $6.06 \pm 2.27 \mathrm{~mJ}$, was higher than in healthy volunteers, $5.47 \pm 2.52 \mathrm{~mJ}$, although this difference was not significant $(P=.65)$ (Figure 2, $A)$. The mean peak systolic $\mathrm{KE}_{\mathrm{LV}}$ in patients with rTOF, $3.55 \pm 2.12 \mathrm{~mJ}$, was also slightly higher than in healthy volunteers, $2.48 \pm 0.75 \mathrm{~mJ}(P=.47)$ (Figure 2, A). When corrected for body surface area (BSA), peak systolic $\mathrm{KE}_{\mathrm{RV}}$ and $\mathrm{KE}_{\mathrm{LV}}$ remained higher in those with $\mathrm{rTOF}\left(3.89 \pm 1.30 \mathrm{~mJ} / \mathrm{m}^{2}\right.$ and $3.55 \pm 2.12 \mathrm{~mJ} / \mathrm{m}^{2}$, respectively) than in healthy volunteers $\left(2.97 \pm 1.36 \mathrm{~mJ} / \mathrm{m}^{2}\right.$ and $2.48 \pm 0.75 \mathrm{~mJ} / \mathrm{m}^{2}$, respectively; $P=.15$ and $P=.08$, respectively). There was no significant difference between BSA-corrected $\mathrm{Q}_{\mathrm{MPA}}(P=.25)$ and $\mathrm{Q}_{\mathrm{AO}}$
$(P=.17)$ between $\mathrm{rTOF}$ and healthy volunteers (Table 2$)$. The mildly higher $\mathrm{Q}_{\mathrm{MPA}}$ and $\mathrm{Q}_{\mathrm{AO}}$ in healthy volunteers compared with rTOF is attributable to a larger scale increase in flow relative to increase in BSA. The $\mathrm{Q}_{\mathrm{MPA}} / \mathrm{KE}_{\mathrm{RV}}$ and $\mathrm{Q}_{\mathrm{AO}} / \mathrm{KE}_{\mathrm{LV}}$ ratios were lower in subjects with rTOF than in healthy volunteers $(P<.05)$ (Figure 2, $B)$. The differences in BSA-corrected $\mathrm{Q}_{\mathrm{MPA}}$ and $\mathrm{Q}_{\mathrm{AO}}$ $(1.3 \times$ and $1.3 \times$ higher in healthy volunteers, respectively) were less compared with the differences in $\mathrm{Q}_{\mathrm{MPA}} / \mathrm{KE}_{\mathrm{RV}}$ and $\mathrm{Q}_{\mathrm{AO}} / \mathrm{KE}_{\mathrm{LV}}$ (2.02 and 2.76 higher in healthy volunteers, respectively). In subjects with rTOF with pulmonary or aortic insufficiency, the $\mathrm{Q}_{\mathrm{MPA}} / \mathrm{KE}_{\mathrm{RV}}$ and $\mathrm{Q}_{\mathrm{AO}} / \mathrm{KE}_{\mathrm{LV}}$ ratios were adjusted to include all forward flow, rather than net flow. These adjusted ratios remained significantly lower in subjects with rTOF than in healthy volunteers $(P<.05)$.

The relationships between $\mathrm{Q}_{\mathrm{MPA}} / \mathrm{KE}_{\mathrm{RV}}$ and $\mathrm{Q}_{\mathrm{AO}} / \mathrm{KE}_{\mathrm{LV}}$ and standard CMR measurements of EDVI, ESVI, and EF are shown in Table 2. Although there was a moderately positive correlation between ventricular-vascular efficiency and RV EDVI and RV ESVI in subjects with rTOF $(r=0.67, P=.03)$, the other comparisons did not reach 
TABLE 2. Summary of standard volumetric and kinetic energy measurements in healthy volunteers and subjects with repaired tetralogy of Fallot

\begin{tabular}{|c|c|c|c|c|}
\hline Mean \pm SD & \multicolumn{2}{|c|}{$\begin{array}{l}\text { Healthy } \\
\text { volunteers }\end{array}$} & rTOF & $P$ values \\
\hline $\operatorname{RVEDVI}\left(\mathrm{mL} / \mathrm{m}^{2}\right)$ & \multicolumn{2}{|c|}{$74 \pm 18$} & $116 \pm 52$ & .03 \\
\hline RVESVI (mL/m²) & \multicolumn{2}{|c|}{$31 \pm 9$} & $60 \pm 27$ & .007 \\
\hline $\operatorname{RVEF}(\%)$ & \multicolumn{2}{|c|}{$58 \pm 8$} & $48 \pm 6$ & .005 \\
\hline $\operatorname{LVEDVI}\left(\mathrm{mL} / \mathrm{m}^{2}\right)$ & \multicolumn{2}{|c|}{$78 \pm 17$} & $71 \pm 20$ & .33 \\
\hline $\operatorname{LVESVI}\left(\mathrm{mL} / \mathrm{m}^{2}\right)$ & \multicolumn{2}{|c|}{$30 \pm 8$} & $31 \pm 12$ & .93 \\
\hline $\operatorname{LVEF}(\%)$ & \multicolumn{2}{|c|}{$62 \pm 3$} & $56 \pm 7$ & .02 \\
\hline $\begin{array}{l}\text { Mean peak systolic } \\
\mathrm{KE}_{\mathrm{RV}}(\mathrm{mJ})\end{array}$ & \multicolumn{2}{|c|}{$5.5 \pm 2.5$} & $6.1 \pm 2.3$ & .65 \\
\hline $\begin{array}{l}\text { Mean peak systolic } \\
\mathrm{KE}_{\mathrm{RV}} / \mathrm{BSA}\left(\mathrm{mJ} / \mathrm{m}^{2}\right)\end{array}$ & \multicolumn{2}{|c|}{$3.0 \pm 1.4$} & $3.9 \pm 1.3$ & .15 \\
\hline $\begin{array}{l}\text { Mean peak systolic } \\
\mathrm{KE}_{\mathrm{LV}}(\mathrm{mJ})\end{array}$ & \multicolumn{2}{|c|}{$2.5 \pm 0.8$} & $3.5 \pm 2.1$ & .47 \\
\hline $\begin{array}{l}\text { Mean peak systolic } \\
\mathrm{KE}_{\mathrm{LV}} / \mathrm{BSA}\left(\mathrm{mJ} / \mathrm{m}^{2}\right)\end{array}$ & \multicolumn{2}{|c|}{$1.4 \pm 0.4$} & $2.2 \pm 1.1$ & .08 \\
\hline Qmpa/BSA (mL/cycle*m²) & \multicolumn{2}{|c|}{$42.4 \pm 9.8$} & $32.4 \pm 26.7$ & .25 (NS) \\
\hline Qao/BSA (mL/cycle*m²) & \multicolumn{2}{|c|}{$45.8 \pm 9.8$} & $34.3 \pm 23.6$ & .17 (NS) \\
\hline $\mathrm{Qmpa} / \mathrm{KE}_{\mathrm{RV}}$ peak/BSA & \multicolumn{2}{|c|}{$19.3 \pm 18.4$} & $7.5 \pm 5.4$ & .015 \\
\hline Qao/KE $\mathrm{KV}_{\mathrm{LV}}$ peak/BSA & \multicolumn{2}{|c|}{$36.0 \pm 7.7$} & $9.7 \pm 6.6$ & .0003 \\
\hline Pearson correlation & $\boldsymbol{R}$ & $\boldsymbol{R}$ & $P$ va & \\
\hline $\mathrm{Qmpa} / \mathrm{KE}_{\mathrm{RV}}$ vs RVEF & 0.46 & 0.06 & .21 (healthy) & 87 (rTOF) \\
\hline $\mathrm{Qmpa} / \mathrm{KE}_{\mathrm{RV}}$ vs RVEDVI & -0.14 & 0.68 & .72 (healthy) & 03 (rTOF) \\
\hline $\mathrm{Qao} / \mathrm{KE}_{\mathrm{LV}}$ vs LVEF & 0.20 & 0.60 & .61 (healthy & 77 (rTOF) \\
\hline Qao/KE $\mathrm{LV}_{\mathrm{LV}}$ vs LVEDVI & 0.53 & 0.09 & .14 (healthy) & 80 (rTOF) \\
\hline
\end{tabular}

$S D$, Standard deviation; $r T O F$, repaired tetralogy of Fallot; RVEDVI, right ventricular end-diastolic volume index; RVESVI, right ventricular end-systolic volume index; $R V E F$, right ventricular ejection fraction; $L V E D V I$, left ventricular end-diastolic volume index; LVESVI, left ventricular end-systolic volume index; $L V E F$, left ventricular ejection fraction; $K E_{R V}$, right ventricular kinetic energy; $B S A$, body surface area; $K E_{L V}$, left ventricular kinetic energy; $Q m p a$, main pulmonary artery flow; NS, nonsignificant; Qao, aortic flow.

statistical significance. In Figure 3, subjects with rTOF were grouped according to normal and abnormal standard RV volumetric measurements and peak $\mathrm{KE}_{\mathrm{RV}}$ was plotted. No significant $\mathrm{KE}_{\mathrm{RV}}$ differences were seen between these subgroups.

\section{DISCUSSION}

In this study, time-resolved $\mathrm{KE}$ in the RV and LV of healthy volunteers and subjects with rTOF was calculated noninvasively using $4 \mathrm{D}$ flow MRI. Although $\mathrm{KE}_{\mathrm{RV}}$ and $\mathrm{KE}_{\mathrm{LV}}$ were higher in subjects with $\mathrm{rTOF}$ compared with healthy volunteers, $\mathrm{Q}_{\mathrm{MPA}}$ and $\mathrm{Q}_{\mathrm{AO}}$ were the same between groups. This reflects the decreased ventricular-vascular efficiency in rTOF for both pulmonary and systemic circulations. These observations may indicate that patients with rTOF have greater energy loss in the RV and LV compared with healthy volunteers without a corresponding ability to generate increased pulmonary or systemic flow. This study helps illustrate the complex relationship between $\mathrm{KE}$ and flow between the ventricles and vasculature, within the $\mathrm{LV}$ and $\mathrm{RV}$, and during systole and diastole in patients with rTOF compared with healthy volunteers.

Previously, accurate measurements of cardiac pressure and energy required invasive methods such as cardiac catheterization, limiting their utility in standard clinical practice. ${ }^{10,19,20}$ The development of 4D flow MRI has provided a noninvasive method of collecting 4D velocity data needed to determine pressure, flow, and energy measurements throughout the cardiac cycle. ${ }^{21}$ Noninvasive measurements of $\mathrm{KE}$ in the $\mathrm{LV}$ and $\mathrm{RV}$ were first described in prior studies using 4D flow acquisitions and were thought to provide a nondirectional measure of the amount of work invested in overcoming the inertia of intracardiac blood during the cardiac cycle. ${ }^{12,22}$ Although KE comprises only approximately $1 \%$ of the total LV work and $6 \%$ of total $\mathrm{RV}$ work at rest, ${ }^{10,22}$ it plays a larger role in the myocardial work under exercise conditions than at rest. ${ }^{10,11}$ Furthermore, over longer time spans any inefficiencies will contribute to excess work and presumably earlier failure.

To our knowledge, this study was the first to measure time-resolved $\mathrm{KE}_{\mathrm{RV}}$ and $\mathrm{KE}_{\mathrm{LV}}$ in subjects with rTOF using 4D flow MRI. Initial studies using MRI to calculate KE during early diastole using a single slice estimated total $\mathrm{KE}_{\mathrm{LV}}$ to be 4 to 5 times higher than the values measured in that single slice. ${ }^{23}$ However, these studies considered only the rotating vortex-like portion of flow without the straight streams of blood flow. Diastolic KE was later quantified in the RV by Fredricksson and colleagues ${ }^{13}$ with 4D flow MRI using calculated pathlines forward and backward in time from the isovolumetric contraction. Subsequently, Carlsson and colleagues ${ }^{12}$ calculated $\mathrm{KE}_{\mathrm{RV}}$ and $\mathrm{KE}_{\mathrm{LV}}$ throughout the cardiac cycle using a method similar to that used in our study. The peak systolic $\mathrm{KE}$ values calculated in our healthy volunteers were $2.48 \pm 0.75 \mathrm{~mJ}$ in the $\mathrm{LV}$ and $5.47 \pm 2.52 \mathrm{~mJ}$ in the RV, whereas Carlsson and colleagues ${ }^{12}$ obtained values of $4.9 \pm 0.4 \mathrm{~mJ}$ in the $\mathrm{LV}$ and $7.5 \pm 0.8 \mathrm{~mJ}$ in the RV. ${ }^{12}$ The lower KE values calculated using our 4D flow MRI technique are probably related to the fact that the peak velocities measured using PC VIPR were lower than those measured using Cartesian-based 4D flow MRI methods used by Carlsson and colleagues ${ }^{12}$ because of temporal blurring and averaging of velocities over the length of the scan. ${ }^{24}$ Because $\mathrm{KE}$ is proportional to the velocity squared, small differences in velocity are magnified when calculating KE values. Future studies using both approaches for 4D flow MRI will be necessary to confirm this. A benefit of using PC VIPR for 4D flow MRI is that it provides a larger field of view and higher spatial resolution than Cartesian approaches in the same scan acquisition time.

Previous research has shown the mortality rate in rTOF increases from $0.27 \%$ per year to $0.94 \%$ per year in the third decade of life, with sudden cardiac death as the major 

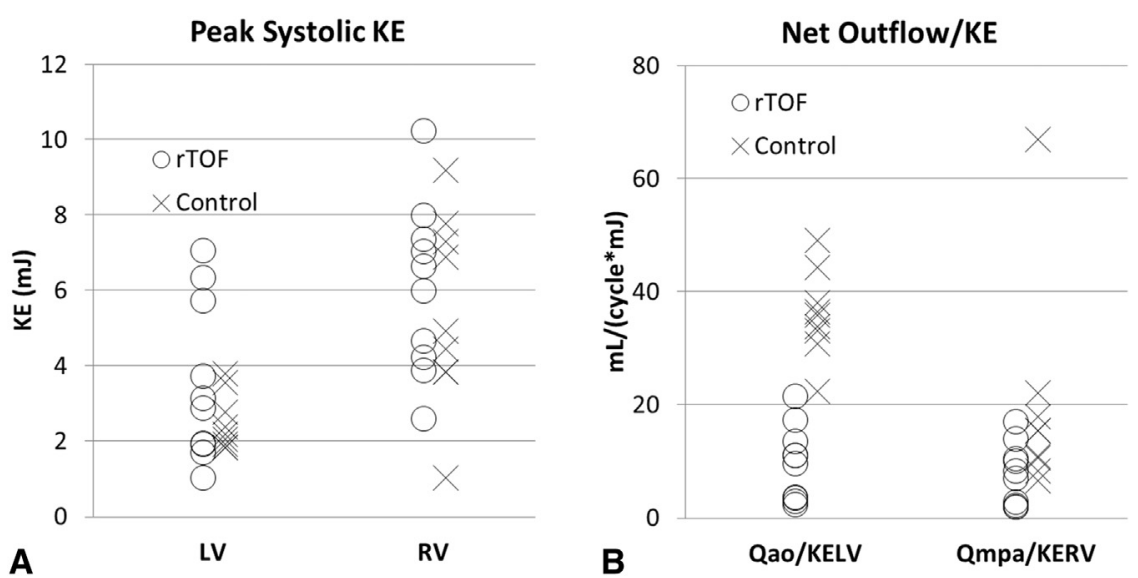

FIGURE 2. A, Peak systolic $\mathrm{KE}_{\mathrm{RV}}$ and $\mathrm{KE}_{\mathrm{LV}}$ comparison between subjects with $\mathrm{rTOF}$ and healthy volunteers. Differences in $\mathrm{KE}_{\mathrm{RV}}(P=.65)$ and $\mathrm{KE} \mathrm{LV}_{\mathrm{LV}}$ $(P=.47)$ are not statistically significant. $\mathrm{B}$, Ratios of outflow to peak systolic ventricular $\mathrm{KE}$ as markers for ventricular-vascular efficiency. $\mathrm{Q}_{\mathrm{MPA}} / \mathrm{KE}_{\mathrm{RV}}$ was significantly lower in rTOF than in healthy volunteers $(P=.015)$. $\mathrm{Q}_{\mathrm{Ao}} / \mathrm{KE}_{\mathrm{LV}}$ was also significantly lower in $\mathrm{rTOF}$ than in healthy volunteers $(P=.0003)$. $K E$, Kinetic energy; $r T O F$, repaired tetralogy of Fallot; $L V$, left ventricular; $R V$, right ventricular; $Q m p a$, main pulmonary artery flow; $K E_{R V}$, right ventricular kinetic energy; $Q a o$, aortic flow; $K E_{L V}$, left ventricular kinetic energy.

cause of death. ${ }^{25}$ It often may be too late to intervene by the time conventional measures such as LVEF have declined, because the patient's risk of sudden cardiac death has already increased. ${ }^{26}$ Even after repair of TOF, research comparing patients with rTOF with healthy subjects found an increased number of vortical flow patterns in the RV, illustrating altered intracardiac flow patterns. ${ }^{7}$ RV-PA coupling has been shown to be impaired in patients with rTOF and proposed as a maladaptive response of the pulmonary arterial system to chronic RV hemodynamic derangements. ${ }^{27}$ Disorganized flows within the ventricle may contribute to KE but not necessarily to ventricular outflow, representing ventricular-vascular inefficiency. Ericksson and colleagues ${ }^{28}$ reported higher $\mathrm{KE}_{\mathrm{LV}}$ in patients with dilated cardiomyopathy than in healthy volunteers despite similar stroke volumes in both groups. Although the ventricular-vascular relationship has been expressed as the ratio between arterial elastance and end-systolic elastance, ${ }^{14}$ we used the relationship between peak systolic KE and ventricular outflow to serve as an indirect measure of ventricular-vascular inefficiency with respect to the blood pool. The range of $\mathrm{Q}_{\mathrm{MPA}} / \mathrm{KE}_{\mathrm{RV}}$ and $\mathrm{Q}_{\mathrm{AO}} / \mathrm{KE}_{\mathrm{LV}}$ efficiency ratios was relatively large in our healthy volunteers, and this could be explained by the small peak systolic KE denominator component of 1.83 to 3.80 $\mathrm{mJ}$ in the $\mathrm{LV}$ and 1.03 to $9.18 \mathrm{~mJ}$ in the RV. Although the KE values and flows were similar throughout healthy volunteers, a wider range of normal values would be expected when the ratio is taken. The rTOF ventricular efficiency ratios had a narrower range due to the higher peak systolic KE values.

Overall, there were no strong correlations between (A) $\mathrm{KE}_{\mathrm{RV}}, \mathrm{KE}_{\mathrm{LV}}, \mathrm{Q}_{\mathrm{MPA}} / \mathrm{KE}_{\mathrm{RV}}$, and $\mathrm{Q}_{\mathrm{AO}} / \mathrm{KE}_{\mathrm{LV}}$ and (B) conventional measurements of ventricular size or function. These results indicate that ventricular $\mathrm{KE}$ and the flow/KE ratios are an earlier indicator of abnormal cardiac function than EDVI, ESVI, and EF. We hypothesize that these altered hemodynamics precede the gross morphologic changes of the myocardium that are quantified with these standard cardiac measurements. Consequently, $\mathrm{KE}$ as a reflection of disordered flow and ventricular inefficiency may provide an earlier indicator of ventricular dysfunction.

Although altered hemodynamics may precede gross morphologic changes in the myocardium, the relationship among KE, hemodynamic derangements, and microscopic myocardial changes warrants further study. Moderate RV or LV systolic dysfunction has been associated with poor clinical status of long-term survivors with rTOF, and reduced LVEF in patients with rTOF has been shown to be one of the strongest predictors of mortality. ${ }^{5,27}$ Further studies have shown correlation between RV and LV systolic dysfunction, suggesting unfavorable ventriculoventricular interactions. ${ }^{27,29}$ Our results demonstrate increased KE and decreased efficiency in both the RV and LV of patients with rTOF. We found higher KE in the LV compared with the RV, which is compatible with previous findings. ${ }^{10,13}$ Of note, Carlsson and colleagues ${ }^{12}$ reported higher peak systolic KE in the RV than the LV. However, the exact mechanisms of how altered hemodynamics and energy might contribute to ventricular dysfunction are unclear. LV contraction contributes to RV pressure development, and RV loading affects LV function, but additional factors such as shared myocardial fibers and septal deviation have been implicated in producing unfavorable interventricular interactions that contribute to ventricular dysfunction in rTOF $^{5,30}$ This ventricular dyssynchrony reduces net cardiac output and reduces 
RVEDVI vs. Ventricular Efficiency

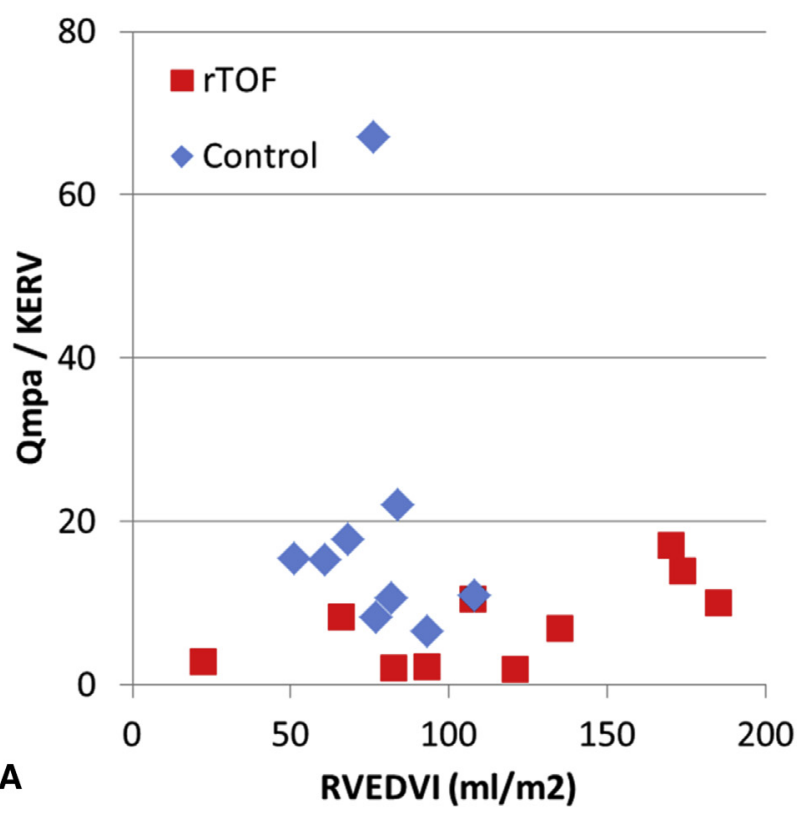

LVEDVI vs. Ventricular Efficiency

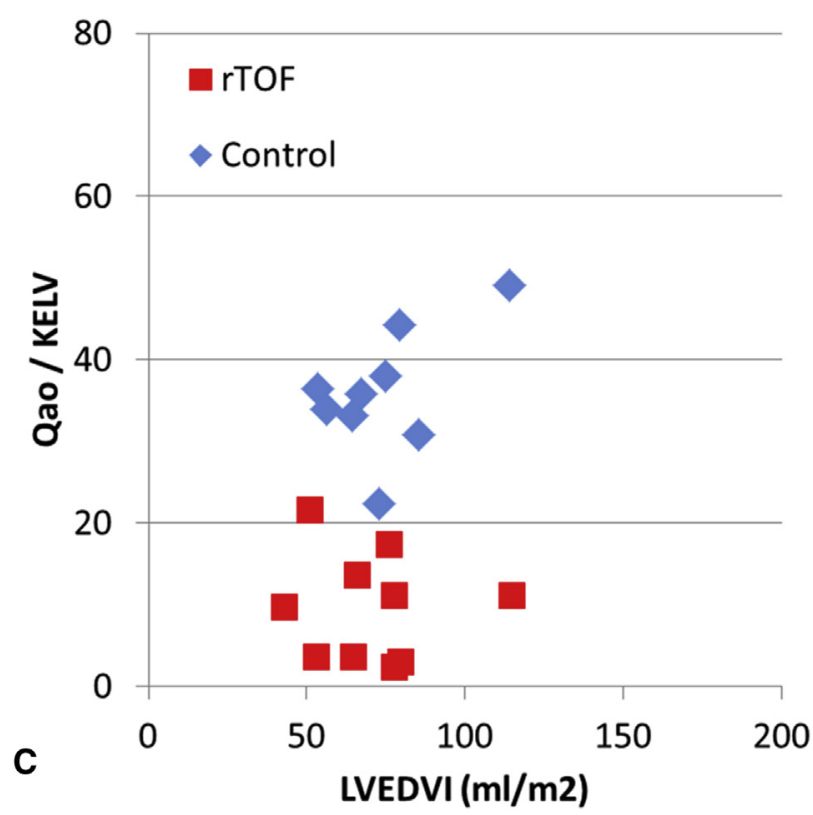

RVEF vs. Ventricular Efficiency

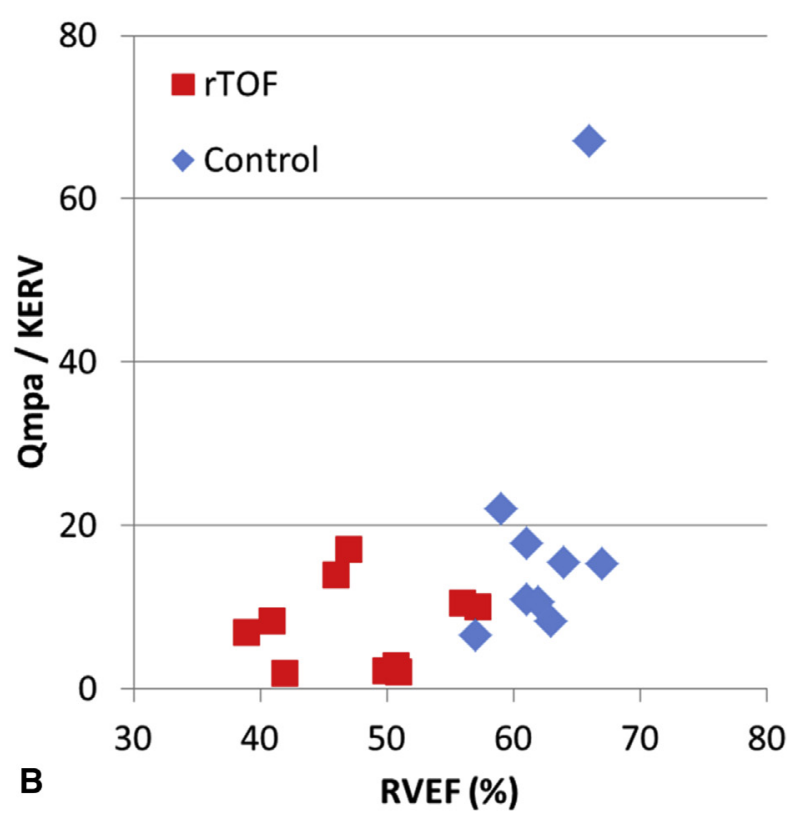

RVEF vs. Ventricular Efficiency

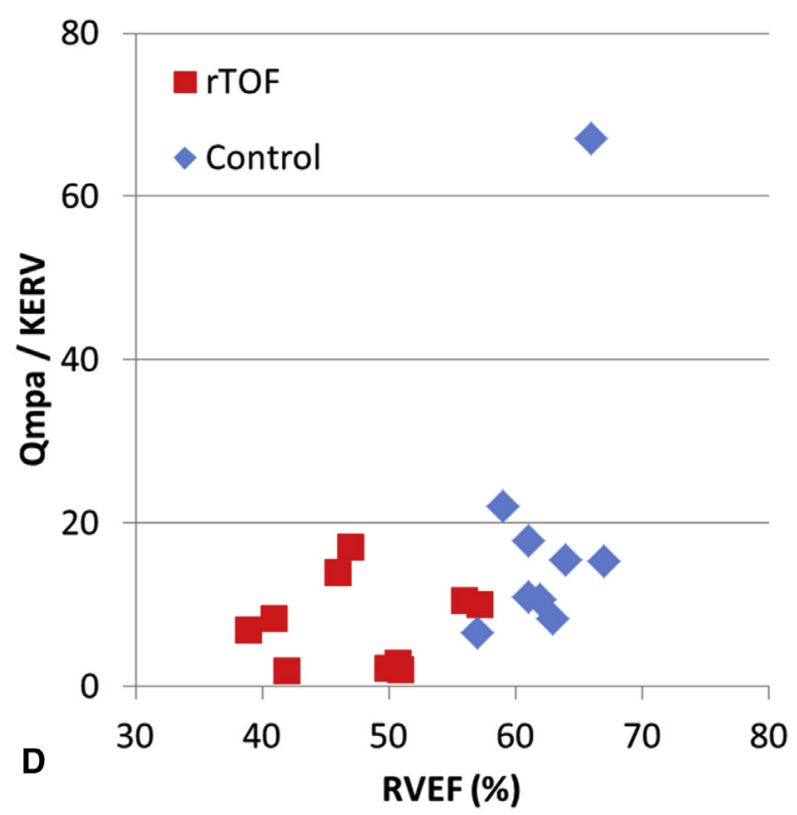

FIGURE 3. A, RVEDVI versus ventricular efficiency. B, RVEF versus ventricular efficiency. C, LVEDVI versus ventricular efficiency. D, LVEF versus ventricular efficiency. There is a moderately positive correlation between ventricular efficiency and RVEDVI in subjects with rTOF $(r=0.68, P=.03$ ) demonstrated by the red boxes in A. However, the other comparisons did not reach statistical significance. $R V E D V I$, Right ventricular end-diastolic volume index; $Q m p a$, main pulmonary artery flow; $K E_{R V}$, right ventricular kinetic energy; $r T O F$, repaired tetralogy of Fallot; $R V E F$, right ventricular ejection fraction; $L V E D V I$, left ventricular end-diastolic volume index; $Q a o$, aortic flow; $K E_{L V}$, left ventricular kinetic energy; $L V E F$, left ventricular ejection fraction.

myocardial efficiency. ${ }^{31}$ Ventricular strain, or local myocardial deformation, evaluated by CMR and echocardiography speckle-tracking may be an earlier predictor of regional ventricular dysfunction compared with $\mathrm{EF}$ in patients with rTOF. ${ }^{3,5,26}$ Whether microscopic myocardial changes or changes in $\mathrm{KE}$ and altered hemodynamics 
provide better indicators for declining ventricular function warrant further investigation.

In addition to examining biventricular $\mathrm{KE}$, we also explored the time-resolved KE changes throughout the cardiac cycle. As in previous studies, ${ }^{12}$ we observed 1 systolic and 2 diastolic KE peaks. Carlsson and colleagues ${ }^{12}$ showed that the $\mathrm{KE}_{\mathrm{RV}}$ was highest along the RV outflow tract during systole and that the $\mathrm{KE}_{\mathrm{RV}}$ was highest from the tip of the tricuspid valve to the center of the ventricle during diastole. Our findings showed a similar distribution of $\mathrm{KE}$ in healthy volunteers throughout the cardiac cycle. In subjects with $r T O F$, higher diastolic $\mathrm{KE}_{\mathrm{RV}}$ was seen along the RV outflow tract because of the presence of locally increased retrograde flow from pulmonic regurgitation.

\section{Study Limitations}

Although the magnitude images from 4D flow MRI have lower contrast between blood-pool and myocardium than steady-state free precession images, Roldan-Alzate and colleagues $^{17}$ reported that ventricular volumes measured using this method are accurate. Using a time average of the magnitude images allowed for a single ventricular segmentation to be performed on images intrinsic to the 4D flow MRI data. An alternative would have been to segment the ventricles on all phases using time-resolved magnitude images. However, this method would be more time-consuming. Using time averaged images in the segmentation would be more limited in the setting of hyperdynamic ventricular motion but would be less limited in the setting of decreased ventricular wall motion.

Some of the subjects with rTOF were scanned at $1.5 \mathrm{~T}$ and others at 3T, whereas all healthy volunteers were scanned at $3 \mathrm{~T}$ with minor differences in parameters between 1.5 and 3T. This introduces a possible source of variability in the data. In addition, 3 subjects with rTOF did not receive IV contrast because of IV access difficulty and poor renal function. Although previous studies have shown that phase-contrast imaging after IV contrast administration improves signal to noise, ${ }^{16}$ adequate phase-contrast imaging was obtainable both before and after contrast.

Other limitations of this study include the small size of the 2 cohorts and heterogeneity of the subjects with rTOF. The study could have been strengthened with age and BSA-matched healthy volunteers, which may have reduced differences in flow data. Further studies in subjects with rTOF with similar repairs and with imaging performed earlier after repair will be necessary to determine the longitudinal significance of quantifying these hemodynamic abnormalities on long-term outcomes. Although we calculated the intraventricular KE, we did not account for the earliest stages of generating ventricular energy at the myocardial level, which other studies have evaluated. ${ }^{31,32}$ Also, the ventricular efficiency ratio proposed in this study used the peak systolic ventricular $\mathrm{KE}$ and aortic or main PA outflow. This was introduced as one marker for ventricular efficiency with respect to KE of the ventricular blood pool. An alternative consideration of ventricular efficiency could include the myocardial work that relates to generating ventricular outflow, which was not directly evaluated in this study. Correlation with pressure-volume loops available through catheterization would offer further insight into the degree of myocardial work that is transferred into KE. Finally, these metrics were all obtained at rest in the supine position. Patients with rTOF often have decreased exercise tolerance, which may be a reflection of inefficient ventricular contractions. During exercise, the flow rates and flow patterns that were observed in this study would likely change with KE playing a larger role.

\section{CONCLUSIONS}

Quantification of increased ventricular KE in patients with rTOF is a new observation. Our study illustrates the complex relationship between ventricular $\mathrm{KE}$ and flow through the great vessels. $\mathrm{KE}_{\mathrm{RV}}$ and $\mathrm{KE}_{\mathrm{LV}}$ were higher and $\mathrm{Q}_{\mathrm{MPA}} / \mathrm{KE}_{\mathrm{RV}}$ and $\mathrm{Q}_{\mathrm{AO}} / \mathrm{KE}_{\mathrm{LV}}$ ratios were lower in patients with rTOF than in healthy volunteers. This is indicative of significantly greater energy losses in the RV and LV in patients with rTOF without a corresponding ability to generate increased pulmonary or systemic flow. Also, KE constitutes a greater portion of the total work in the right side of the heart than in the left side of the heart at baseline and during activity. Future studies may determine a closer relationship of $\mathrm{KE}_{\mathrm{RV}}$ and $\mathrm{KE}_{\mathrm{LV}}$ with exercise performance and capacity than standard CMR measurements. Although KE measurements derived from 4D flow MRI provide a novel noninvasive method of monitoring cardiac function, future investigations will be needed to determine whether changes in ventricular KE can provide earlier evidence of ventricular dysfunction and guide future medical and surgical interventions.

\section{Conflict of Interest Statement}

The University of Wisconsin receives research support from GE Healthcare. All other authors have nothing to disclose with regard to commercial support.

\section{References}

1. Starr JP. Tetralogy of Fallot: yesterday and today. World J Surg. 2010;34 658-68.

2. Lloyd-Jones D, Adams RJ, Brown TM, Carnethon M, Dai S, De Simone G, et al. Heart disease and stroke statistics-2010 update: a report from the American Heart Association. Circulation. 2010;121:e46-215.

3. Zhong L, Gobeawan L, Su Y, Tan J, Ghista D, Chua T, et al. Right ventricular regional wall curvedness and area strain in patients with 
repaired tetralogy of Fallot. Am J Physiol Heart Circ Physiol. 2012;302: H1306-16.

4. Adamson L, Vohra HA, Haw MP. Does pulmonary valve replacement post repair of tetralogy of Fallot improve right ventricular function? Interact Cardiovasc Thorac Surg. 2009;9:520-7.

5. Khalaf A, Tani D, Tadros S, Madan S. Right- and left- ventricular strain evaluation in repaired pediatric tetralogy of Fallot patients using magnetic resonance tagging. Pediatr Cardiol. 2013;34:1206-11.

6. Therrien J, Provost Y, Merchant N, Williams W, Colman J, Webb G. Optimal timing for pulmonary valve replacement in adults after tetralogy of Fallot repair. Am J Cardiol. 2005;95:779-82.

7. Francois CJ, Srinivasan S, Schiebler ML, Reeder SB, Niespodzany E, Landgraf BR, et al. 4D cardiovascular magnetic resonance velocity mapping of alterations of right heart flow patterns and main pulmonary artery hemodynamics in Tetralogy of Fallot. J Cardiovasc Magn Reson. 2012; $14: 16$.

8. Geiger J, Markl M, Jung B, Grohmann J, Stiller B, Langer M, et al. 4D-MR flow analysis in patients after repair for tetralogy of Fallot. Eur Radiol. 2011;21: 1651-7.

9. Harrild DM, Berul CI, Cecchin F, Geva T, Gauvreau K, Pigula F, et al. Pulmonary valve replacement in tetralogy of Fallot: impact on survival and ventricular tachycardia. Circulation. 2009;119:445-51.

10. Prec O, Katz N, Sennett L, Rosenman RH, Fishman AP, Hwang W. Determination of kinetic energy of the heart in man. Am J Physiol. 1949;159:483-91.

11. Rao PS, Awa S, Linde LM. Role of kinetic energy in pulmonary valvar pressure gradients. Circulation. 1973;48:65-73.

12. Carlsson M, Heiberg E, Toger J, Arheden H. Quantification of left and right ventricular kinetic energy using four-dimensional intracardiac magnetic resonance imaging flow measurements. Am J Physiol Heart Circ Physiol. 2012;302: H893-900.

13. Fredricksson AG, Zajac J, Eriksson J, Dyverfeldt P, Bolger AF, Ebbers T, et al. 4D blood flow in the human right ventricle. Am J Physiol Heart Circ Physiol. 2011;301:H2344-50.

14. Antonini-Canterin F, Poli S, Vriz O, Pavan D, Bello VD, Nicolosi GL. The ventricular-arterial coupling: from basic pathophysiology to clinical application in the echocardiography laboratory. J Cardiovasc Echogr. 2013;23: $91-5$.

15. Gu T, Korosec FR, Block WF, Fain SB, Turk Q, Lum D, et al. PC VIPR: a high-speed 3D phase-contrast method for flow quantification and high-resolution angiography. Am J Neuroradiol. 2005;26:743-9.

16. Bock J, Frydrychowicz A, Stalder AF, Bley TA, Burkhardt H, Hennig J, et al. 4D phase contrast MRI at 3 T: effect of standard and blood-pool contrast agents on SNR, PC-MRA, and blood flow visualization. Magn Reson Med. 2010;63:330-8.

17. Roldan-Alzate A, Jensen L, Frydrychowicz A, Nagle SK, Kellihan H, Chesler N, et al. Free breathing measurement of ventricular volumes from magnitude data of 4D flow-sensitive MRI in a canine model of acute pulmonary arterial hypertension [abstract]. In Proceedings of the 20th Annual Meeting of the International
Society for Magnetic Resonance in Medicine, May 5-11, 2012, Melbourne Australia.

18. Hinghofer-Szalkay H, Greenleaf JE. Continuous monitoring of blood volume changes in humans. J Appl Physiol. 1987;63:1003-7.

19. Milnor WR, Bergel D, Bargainer JD. Hydraulic power associated with pulmonary blood flow and its relation to heart rate. Circ Res. 1966;19: 467-80.

20. O'Rourke MF. Impact pressure, lateral pressure, and impedance in the proximal aorta and pulmonary artery. J Appl Physiol. 1968;25:533-41.

21. Lee N, Taylor MD, Hor KN, Banerjee RK. Non-invasive evaluation of energy loss in the pulmonary arteries using $4 \mathrm{D}$ phase contrast MR measurement: a proof of concept. Biomed Eng Online. 2013;12:93.

22. Arvidsson PM, Toger J, Heiberg E, Carlsson M, Arheden H. Quantification of left and right atrial kinetic energy using four-dimensional intracardiac magnetic resonance imaging flow measurements. J Appl Physiol. 2013;114 $1472-81$.

23. Kim WY, Walker PG, Pedersen EM, Poulsen JK, Oyre S, Houlind K, et al. Left ventricular blood flow patterns in normal subjects: a quantitative analysis by three-dimensional magnetic resonance velocity mapping. J Am Coll Cardiol. 1995;26:224-38

24. Wentland AL, Grist TM, Wieben O. Repeatability and internal consistency of abdominal 2D and 4D phase contrast MR flow measurements. Acad Radiol. 2013;20:699-704.

25. Nollert G, Fischlein T, Bouterwek S, Bohmer C, Klinner W, Reichart B. Long-term survival in patients with repair of tetralogy of Fallot: 36-year follow-up of 490 survivors of the first year after surgical repair. J Am Coll Cardiol. 2002;30:1374-83.

26. Ordovas KG, Carlsson M, Lease KE, Foster E, Meadows AK, Martin AJ, et al Impaired regional left ventricular strain after repair of tetralogy of Fallot. J Magn Reson Imaging. 2012;35:79-85.

27. Geva T, Sandweiss BM, Gauvreau K, Lock JE, Powell AJ. Factors associated with impaired clinical status in long-term survivors of Tetralogy of Fallot repair evaluated by magnetic resonance imaging. J Am Coll Cardiol. 2004;43: 1068-74.

28. Ericksson J, Bolger AF, Ebbers T, Carlhall CJ. Four-dimensional blood flow-specific markers of LV dysfunction in dilated cardiomyopathy. Eur Heart J Cardiovasc Imaging. 2013;14:417-24.

29. Kempny A, Diller G, Orwat S, Kaleschke G, Kerckhoff G, Bunck AC, et al Right ventricular-left ventricular interaction in adults with Tetralogy of Fallot: a combined cardiac magnetic resonance and echocardiographic speckle tracking study. Int J Cardiol. 2012;154:259-64.

30. Friedberg MK, Redington AN. Right versus left ventricular failure: differences, similarities, and interactions. Circulation. 2014;129:1033-44.

31. Cheng A, Helm R, Abraham TP. Pathophysiological mechanisms underlying ventricular dyssynchrony. Europace. 2009;11(Suppl 5):v10-4.

32. Granzier HL, Labeit S. The giant protein titin: a major player in myocardial mechanics, signaling, and disease. Circ Res. 2004;94:284-95. 